\title{
SMART CV FOR LIFELONG QUALIFICATIONS CERTIFICATION BASED ON BLOCKCHAIN
}

\author{
Anastasios A. Economides, \& Maria Perifanou \\ SMILE lab, University of Macedonia (Greece)
}

\begin{abstract}
There is a need for fast, automatic, and trusted verification of a person's qualifications. This paper proposes the Smart CV (Curriculum Vita) that contains links to Blockchain-based certifications of the person's qualifications. Also, the paper proposes an architecture for the Blockchain-based Smart CV consisting of five layers: 1) Trusted certificate Issuers; 2) Trusted Distributed Ledgers; 3) Trusted management; 4) Smart CV; 5) Users. Educational institutes, accreditation organizations, public authorities, employers and others can cooperate to issue and accept these Blockchain-based certificates presented on a person's Smart CV.
\end{abstract}

Keywords: Accreditation, blockchain, certificates, lifelong education, qualifications certification.

\section{Introduction}

Currently, there are serious ethical problems in higher education with regards to copying and fake degrees (Børresen, et al., 2020). Most educational certificates are issued and stored as digital certificates, and they face security dangers and adulteration. Employers, teachers, university administrators, and other officials have serious concerns about the validity of these certificates and they do not trust them. In addition, when employers and recruiting agencies screen candidates' CVs (Curriculum Vita), they would like to fast and easily verify these candidates' qualifications. Therefore, there is a need for an automatic, trusted, open, and transparent solution for managing and verifying qualifications. This paper proposes the use of the Blockchain technology for qualifications' certification in education (formal, informal, and non-formal), employment, and other cases. It proposes a Smart CV linked with Blockchain-based certifications of the information included in it. It also proposes a model for the Smart CV as well as an architecture for the interactions of all entities involved (e.g., certificates' providers, public authorities, professional licenses providers, educational institutes, employers, persons) and the Distributed Pledges.

The blockchain is a security technology that has been applied in many sectors including education (Sharples \& Domingue, 2016). Blockchain technology can create new opportunities and challenges for knowledge and skills certification anytime and anyplace. It provides a decentralised trusted peer-to-peer infrastructure, which also supports identity management, openness, transparency, and accountability (Mikroyannidis et al., 2019). The blockchain is a long chain of linked blocks securely stored on every participating computer. Each block typically contains a link to a previous block, a timestamp and transaction data. The next block can only be added if the majority of participants agree on.

Blockchain technology has been used by various secure issuing and verification protocols. These protocols store metadata regarding the certificate issuer, the data issued, and the type of certificate. Blockcerts (MIT, 2020) is an open standard for creating, issuing, viewing, and verifying blockchain-based certificates. Individuals are able to possess and share their own official records. Arizona State University's Trusted Learner Network (TLN) (ASU, 2019) is a decentralized approach to securely recording, curating, and sharing learner's data. There are 12 principles of the TLN: 1) Stores learner achievements; 2) Safe and secure; 3) Always up-to-date; 4) Issued and maintained by the entity; 5) Presents current information first; 6) Records learner consent; 7) Shared only within the TLN; 8) Co-owned by the learner; 9) Open-source tools and APIs; 10) Identity and access management tools included; 11) Non-commercial use; 12) Network of networks. 
A certificate issuer (e.g., Universities, Certification and License providers) can add a block containing a certificate for a person in the blockchain. The data about the certificate are timestamped and securely stored in all participating computers. Anyone (e.g., the person, a teacher, an employer) having the cryptographic 'public key' can view these data. However, no one can change these data, even the original certificate issuer. The University of Nicosia used Bitcoin blockchain to issue and verify academic certificates (UNIC, 2020). Also, Sony Global Education (SGE) developed a blockchain-based system to record and reference educational data and digital transcripts from multiple educational institutions (SONY, 2017). SAP introduced TrueRec, a Blockchain-based digital wallet for storing professional and academic credentials (SAP, 2017). Whenever a new credential is issued through TrueRec, the user receives the credential and its digital fingerprint is recorded on the blockchain. When the user sends this credential to someone, the receiver can instantly verify its validity.

Rooksby \& Dimitrov (2017) developed an Ethereum Blockchain-based system to store student grades and to provide a cryptocurrency. Also, Skiba (2017) developed a Blockchain-based secure and trustworthy system to allow students to own their credentials from different universities. Similarly, Turkanović et al. (2018) as well as Gräther et al. (2018) developed platforms for certificate issuing, validating, and sharing. Furthermore, Mikroyannidis et al. (2019) proposed a Blockchain-based personal Learning Passport that contains Smart Badges from both formal and informal learning organizations.

This paper extends the traditional digital CV exploiting the Blockchain technology. In a CV, a person lists titles of his/her experiences and certificates acquired from various institutes, organizations, businesses, and employers. However, there is no guarantee for the accuracy of all these information. Blockchain technology can verify the integrity of a certificate, its timestamp, its issuer, and much more.

First, this paper introduces a Smart CV model. Then it introduces an architecture for all entities involved. Finally, it concludes and suggests future research directions.

\section{Smart CV}

A person's CV includes information regarding certificates issued by public and private authorities. These certificates include licenses, degrees, credentials, records, credits, test results, badges, awards, positions, experience, endorsements, recommendation letters, etc. A certificate is a proof (verification) that the person successfully passed specific thresholds or achieved specific outcomes or completed specific requirements. For example, a certificate may certify that the person participated in an activity above a specific level (e.g., above $80 \%$ attendance in the activity, or above $60 \%$ completed assignments in the activity), or that the person successfully passed a specific threshold in a specific activity (e.g., test, exam, work), or achieved a specific score, outcome, achievement, artifact, award, professional status, position, etc.

This paper defines Smart CV to be a digital CV containing the certifications for any information reported in it. In the same way a digital document may include web links to information in the Web, a smart CV include links to the corresponding certificates in one or more Blockchains. The validity of the Smart CV is provided by the Blockchain.

The next Figure 1 shows a Smart CV. For example, the certificate "DS Training Cert1" confirms that the person has "successfully" participated in the Digital Skills Training \#1 (e.g., Python programming). In parallel, the certificates "DS Cert1" and "DS Cert2" confirm that the person has successfully passed the exams for the Digital Skills certification \#1 (e.g., Certified Entry-Level Python Programmers- PCEP), and the Digital Skills Certification \#2 (e.g., Microsoft Technology Associate 98-381: Introduction to Programming with Python). All these certifications are officially issued by the appropriate authorities. In the Smart CV, there are links to the corresponding data in the Blockchains. Anyone holding the public key for a specific certificate can see it. So, the Smart CV owner can grant access to specific certificates to specific recipients.

Further granularity can be also incorporated in this Smart CV model. For example, the Smart CV can include the courses taken for the M.Sc. degree and all work done for each course (Figure 2). Also, it can include certificates for professional responsibilities and achievements in various jobs.

Finally, let us define an Open Smart CV. An Open Smart CV is a Smart CV that is Open to anyone, i.e., all information and certificates listed in this Smart CV can be accessed and viewed by anyone. In a partially Open Smart CV, only specific information and certificates are open to specific people or machines. The degree of its Openness depends on how much information is open to how many people or machines. 
Figure 1. An example of a Smart $C V$.

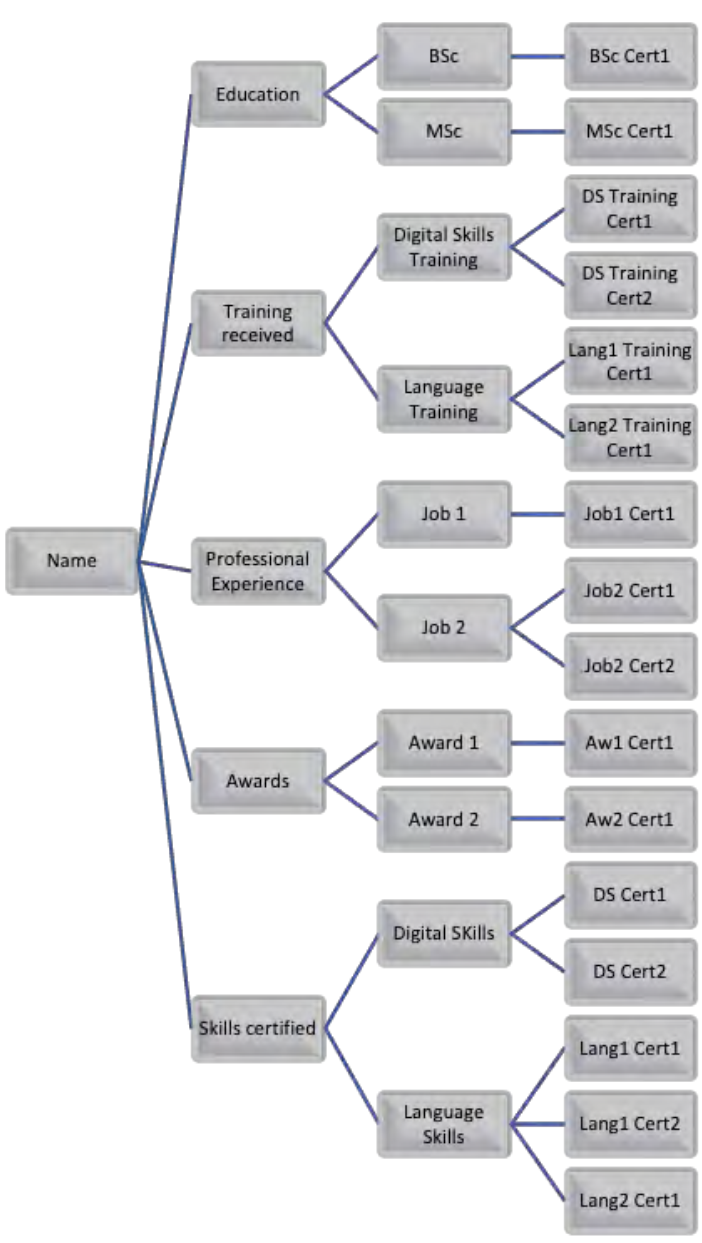

Figure 2. More granularity of a Smart CV example.

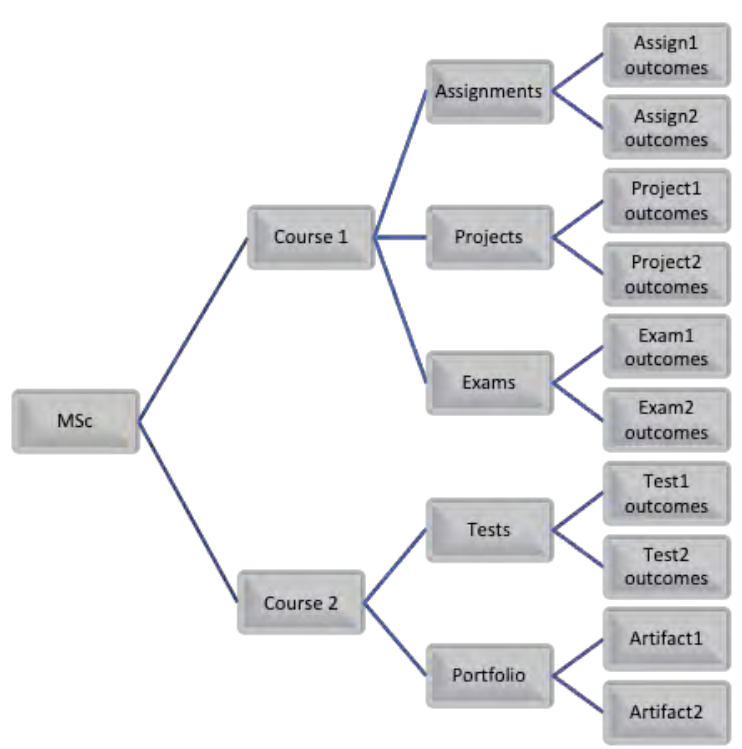

\section{Architecture}

In this section, the paper introduces an architecture for the Blockchain-based Smart CV (Figure 3). There are 5 layers in this architecture: 1) Trusted Certificate Issuers; 2) Trusted Distributed Ledgers; 3) Trusted Management; 4) Smart CV; 5) Users. 
Figure 3. Architecture of the Blockchain-based Smart CV.

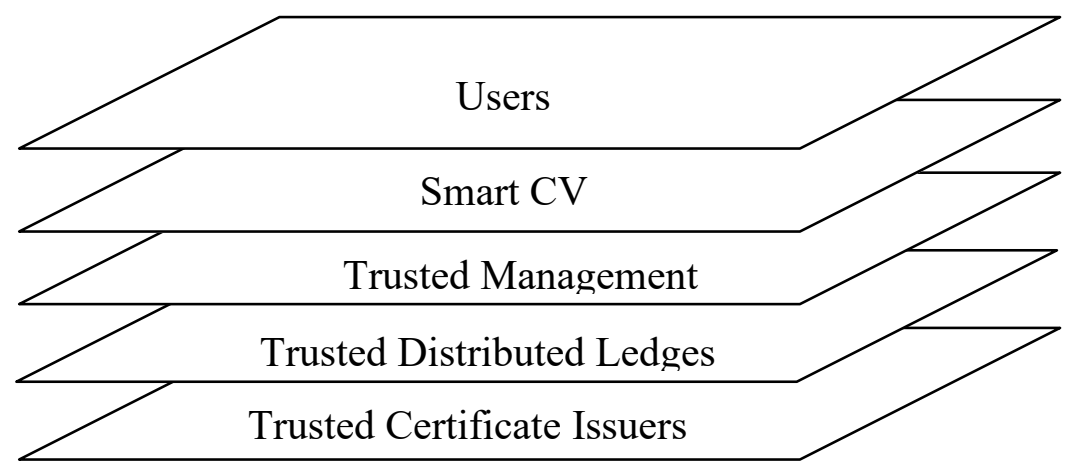

A Certificate Issuer can be any public or private authority that issues certificates (e.g., educational institute, accreditation or certification organization, employer, government authority, professional association, licensing authority). A User can be the person itself (the smart CV owner), any Certificate Issuer, and anyone who is interested in evaluating the person's Smart CV (e.g., educational institute, teacher, employer).

Figure 4. Interactions among Certificate Issuers and Certificate Users.

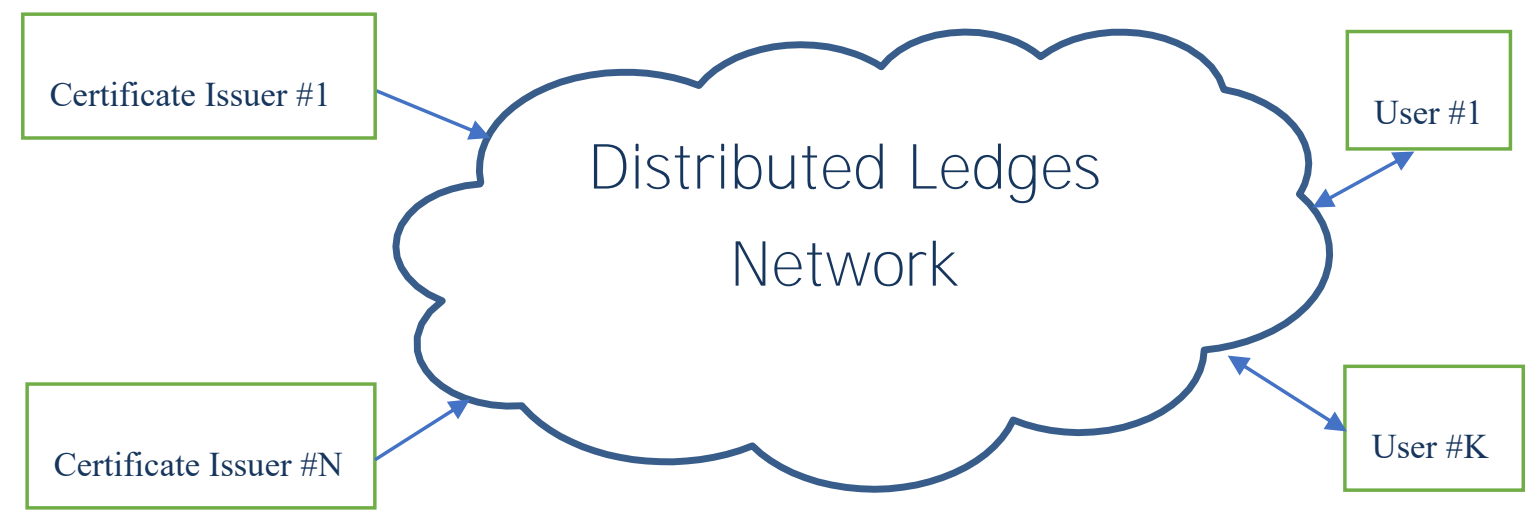

\section{Conclusions and future research}

Recently, there is an increase of fraud in the area of qualifications certifications. Fake certifications are submitted by candidate employees and students applying for a position, a scholarship or job. This paper proposes the Smart CV to fight this problem. A certification authority issues a certificate which is stored in a Blockchain. The Smart CV contains a link to this securely stored certificate. Anyone holding the public key for this specific certificate can see it. Educational institutes, accreditation organizations, public authorities, employers and others can cooperate to issue and accept these Blockchain-based certificates. The paper also provides an Architecture of the Blockchain-based Smart $\mathrm{CV}$ and the Interactions among Certificate Issuers and Certificate Users. Future research may try to implement this Smart CV with rear data.

\section{References}

ASU (2019). The Trusted Learner Network (TLN). Retrieved 12-12-2020 from: https://uto.asu.edu/initiatives/trusted-learner-network

Børresen, L. J., Meier, E., \& Skjerven, S. A. (2020). Corruption in higher education. Global challenges and responses. Series: Global Perspectives on Higher Education, Volume: 46, Brill Publ.

Chapman, D. W., \& Lindner, S. (2016). Degrees of integrity: The threat of corruption in higher education. Studies in Higher Education, 41(2), 247-268. 
Gräther, W., Kolvenbach, S., Ruland, R., Schütte, J., Torres, C., \& Wendland, F. (2018). Blockchain for education: Lifelong learning passport. In Proceedings of 1st ERCIM Blockchain Workshop 2018. European Society for Socially Embedded Technologies (EUSSET).

Grech, A., Camilleri, A.F., \& Inamorato dos Santos, A. (2017). Blockchain in education. Publications Office of the European Union, Luxembourg. ISBN 978-92-79-73497-7. doi: 10.2760/60649

Harris, J., \& Wihak, C. (2017). To what extent do discipline, knowledge domain and curriculum affect the feasibility of the Recognition of Prior Learning (RPL) in higher education? International Journal of Lifelong Education, 36(6), 696-712.

Kontzinos, C., Kokkinakos, P., Skalidakis, S., Markaki, O., Karakolis, V., \& Psarras, J. (2020). Decentralised qualifications' verification and management for learner empowerment, education reengineering and public sector transformation: The QualiChain project. Mobile, Hybrid, and On-line Learning (eLmL 2020), 51.

Lundvall, B. Å., \& Rasmussen, P. (2016). Challenges for adult skill formation in the globalising learning economy-a European perspective. International Journal of Lifelong Education, 35(4), 448-464.

Mayombe, C. (2017). An assessment of non-formal education and training centres' linkages with role-players for adult employment in South Africa. International Journal of Lifelong Education, 36(3), 339-358.

Mikroyannidis, A., Domingue, J., Bachler, M., \& Quick, K. (2018). Smart blockchain badges for data science education. In: 2018 IEEE Frontiers in Education Conference (FIE) (pp. 1-5). IEEE.

Mikroyannidis, Al., Third, Al., \& Domingue, J. (2019). Decentralising online education using blockchain technology. In: The Online, Open and Flexible Higher Education Conference: Blended and online education within European university networks, 16-18 Oct 2019, UNED, Madrid.

MIT (2020). Blockcerts - The Open Standard for Blockchain Credentials. Retrieved 12-12-2020 from: https://www.blockcerts.org/

Mohamedbhai, G. (2016). The scourge of fraud and corruption in higher education. International Higher Education, (84), 12-14. https://doi.org/10.6017/ihe.2016.84.9111

Müller, R., Remdisch, S., Köhler, K., Marr, L., Repo, S., \& Yndigegn, C. (2015). Easing access for lifelong learners: a comparison of European models for university lifelong learning. International Journal of lifelong education, 34(5), 530-550.

Rooksby, J., \& Dimitrov, K. (2019). Trustless education? A blockchain system for university grades. Ubiquity: The Journal of Pervasive Media, 6(1), 83-88.

SAP (2017). Meet TrueRec by SAP: Trusted digital credentials powered by Blockchain. Retrieved 12-12-2020 from: https://news.sap.com/2017/07/meet-truerec-by-sap-trusted-digital-credentialspowered-by-blockchain/

Sharples, M., \& Domingue, J. (2016). The blockchain and kudos: A distributed system for educational record, reputation and reward. In: European conference on technology enhanced learning (pp. 490-496). Springer, Cham.

Skiba, D. J. (2017). The potential of blockchain in education and health care. Nursing education perspectives, 38(4), 220-221.

SONY (2017). Sony develops system for authentication, sharing, and rights management using blockchain technology. Retrieved 12-12-2020 from: https://www.sonyged.com/2017/08/10/news/press-blockchain

Turkanović, M., Hölbl, M., Košič, K., Heričko, M., \& Kamišalić, A. (2018). EduCTX: A blockchain-based higher education credit platform. IEEE Access, 6, 5112-5127.

UNIC (2020). Blockchain Initiative: MSc in Digital Currency. Retrieved 12-12-2020 from: https://digitalcurrency.unic.ac.cy/about-the-program/ 